\title{
Confirm AJCC/TNM Cancer Staging Edition
}

National Cancer Institute

\section{Source}

National Cancer Institute. Confirm A/CC/TNM Cancer Staging Edition. NCI Thesaurus.

Code C157095.

A directive to confirm the relevant edition of the AJCC/T NM Cancer Staging Edition. 